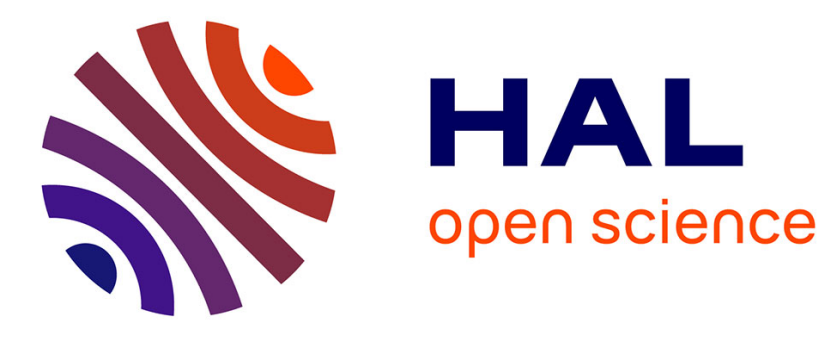

\title{
A biomechanical model of the female reproductive system and the fetus for the realization of a childbirth virtual simulator
}

Romain Buttin, Florence Zara, Behzad Shariat Torbaghan, Tanneguy Redarce

\section{- To cite this version:}

Romain Buttin, Florence Zara, Behzad Shariat Torbaghan, Tanneguy Redarce. A biomechanical model of the female reproductive system and the fetus for the realization of a childbirth virtual simulator. 31st IEEE EMBC, Sep 2009, Minneapolis, MN, United States. pp.5263-5266, 10.1109/IEMBS.2009.5334085 . hal-00507108

\section{HAL Id: hal-00507108 \\ https://hal.science/hal-00507108}

Submitted on 31 Mar 2017

HAL is a multi-disciplinary open access archive for the deposit and dissemination of scientific research documents, whether they are published or not. The documents may come from teaching and research institutions in France or abroad, or from public or private research centers.
L'archive ouverte pluridisciplinaire HAL, est destinée au dépôt et à la diffusion de documents scientifiques de niveau recherche, publiés ou non, émanant des établissements d'enseignement et de recherche français ou étrangers, des laboratoires publics ou privés. 


\title{
A Biomechanical Model of the Female Reproductive System and the Fetus for the realization of a Childbirth Virtual Simulator
}

\author{
R. Buttin ${ }^{1,2}$ and F. Zara ${ }^{1}$ and B. Shariat ${ }^{1}$ and T. Redarce ${ }^{2}$
}

\begin{abstract}
Our main work consists in modeling of the female pelvis and uterus, as well as the human fetus. The goal of this work is to recover the different forces generated during the delivery. These forces will be input to the haptic obstetric training tool BirthSim which has already been developed by the Ampère Laboratory at the INSA of Lyon. This modeling process will permit us to develop a new training device to take into account different anatomies and different types of delivery.

In this paper, we will firstly show the different existing haptic and virtual simulators in the obstetric world with their advantages and drawbacks. After, we will present our approach based on a biomechanical modeling of concerned organs. To obtain interactive time performance, we proceed by the simplification of the organs anatomy. Then, we present some results showing that FEM analysis can be used to model forces during childbirth. In the future, we plan to use this work to more accurately control a childbirth simulator.
\end{abstract}

\section{INTRODUCTION}

Generally, residents learn medical gesture by conducting experiments on real cases. It is the experts' responsibility to guide and transmit their knowledge to the novice trainee. In addition, this learning can result in invasive medical procedures that could threaten patient safety. The use of medical simulators permits the practitioner to acquire some experience before working on real cases. This training method is already used in aerospace or aeronautics [11] for its risk free aspect. In the medical field, some companies propose simulators in ophthalmology, laparoscopy and surgical endoscopy, orthopedic ([3], [6], [5], [8], [16]). In obstetric, very few complete training systems exist.

Our main interest is to develop a virtual simulator for the training of the young obstetricians. In this paper, we present our biomechanical model of the uterus that will be integrated in the whole model of the woman pelvis. In the future, the forces generated during the delivery will be integrated in a haptic system.

In this paper, we first introduce a state of the art of the childbirth simulators. Then, we show briefly the anatomy of female reproductive system and we present our biomechanical approach.

\section{EXISTING CHILDBIRTH SIMULATORS}

It exists two kinds of medical training simulators: the static simulator, and those inspired from the augmented reality techniques. The first type is based on a haptic interface,

\footnotetext{
${ }^{1}$ Université de Lyon, CNRS, Université Lyon 1, LIRIS, SAARA team, UMR5205, F-69622, France firstname.namedliris.cnrs.fr 2 Université de Lyon, CNRS, INSA de Lyon, Laboratoire Ampère, UMR5005, F-69621, France tanneguy.redarcedinsa-lyon. fr
}

composed of several physical (plastic) parts which represent the anatomy of some concerned organs (generally the pelvis and the head of the fetus), as well as a motorized articulated system animating the physical parts and simulating the interaction of the fetus with maternal body and the obstetrician. Thus, a position and force feedback control of motors deriving the articulations will generate resistant forces to reproduce a sensation similar to that felt by the practitioner during the delivery. Moreover, these simulators permit the practitioner to have a very good immersion because of the similarities between anatomical representation by plastic parts and the reality. In this case, the real problem is the development of the haptic device and the realization of the necessary control system to derive the motors. The main disadvantage of this type of devices is that, it is a static system that does not take into account different female morphologies, and/or configurations and conditions related to childbirth. Moreover, the device behavior based on the "sensation felt" by the practitioner could be unrealistic despite some realistic sensations felt. In this category, some childbirth simulators are developed like the GeburtenSimulator of the Automatic Control Laboratory of Zurich [13] or the BirthSim [15] simulator designed in the Ampère Laboratory in Lyon.

The second type of simulators use augmented reality techniques and propose a physically based modeling of the environment. Interactions with the environment through haptic interfaces will provide more realistic effort computations. Thus, with the help of the modeling process, various scenarios reflecting various childbirth configurations could be considered. This compensates the morphology nonadaptability shortcomings of the first type of simulators.

A virtual simulator has already been developed by Boissonnat [4] but they simplify their model by imposing a theoretical fetus trajectory, and the fetal displacement is not the result of a realistic simulation. Kheddar [9] proposed in 2004 another virtual simulator based on a biomechanical model. However, he doesn't consider the uterus as a deformable object but only as a boundary condition. Moreover, like the Boissonnat simulator a theoretical fetus trajectory is imposed.

In the next section, we present a short anatomical study of the female reproductive system, before introducing our approach coupling this two kind of simulators.

\section{ANATOMIC STUDY}

Our goal is to simulate the delivery and to integrate the result of the computed forces generated during childbirth 
into a training simulator. First, we have to understand the role of the different organs and analyze which organs play a significant role (the "useful" and the "fundamental" ones).

Fundamental organs, like the bony pelvis, the fetus, the uterus or the pelvis muscles, play a significant role during the childbirth and need to be modeled more precisely. Indeed, they have a direct role in the determination of the expulsion forces and the trajectory of the fetus. Pelvis muscles, for example, are fixed to the pelvis, and their role is to prevent the descent of the fetus before the pregnancy is "at term". A good geometrical model of the pelvis is also indispensable because this bone interacts with the fetus and guides its trajectory. The last fundamental organ is the womb, a muscular chamber in which the fetus grows during gestation. Moreover, during the delivery, womb contractions move the fetus toward the cervix, causing its dilatation.

The useful organs have not a direct impact on the delivery, but their influence on the delivery process are not insignificant. So, we also take them into account. In this type of organs, we can find the placenta (which brings to the fetus food and oxygen), the bladder and the rectum, some big organs which are directly in contact with the womb. The mechanical properties of these object have an influence on the displacement of the fetus (see Fig 1).

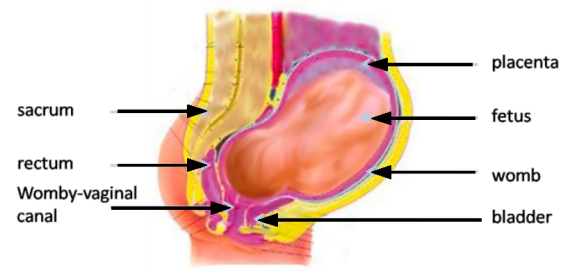

Fig. 1. Anatomy of the pregnant woman

To sum up, we have seen that the forces are caused by the womb. But these uterine contractions are not strong enough to provoke the delivery. Indeed, we also have to consider the action of the abdominal muscles of the mother which push the fetus through out of the birth canal.

\section{OUR BIOMECHANICAL MODEL}

In this section, we propose a biomechanical model of the female reproductive system and the fetus, to simulate the delivery forces to be input into the BirthSim simulator developed in the Ampère Laboratory [10]. This simulator is composed of three parts (see Fig. 2):

- A Graphic User Interface (GUI) used to display the position of the fetal head inside of the pelvis and the different parameters like uterine contraction forces, orientation of the head, or the mother push forces.

- An electro pneumatic system with a pneumatic actuator and a rotating system to simulate the forces and the displacement of the head.

- A mechanical part (haptic interface) which is composed of a plastic representation of the pelvis and the fetal head. This part allows the practitioner to have an interaction with the pneumatic system (haptic interface).
So, our virtual simulator will replace the GUI part of the BirthSim simulator and moreover, will compute the forces which are then generated by the pneumatic actuators.

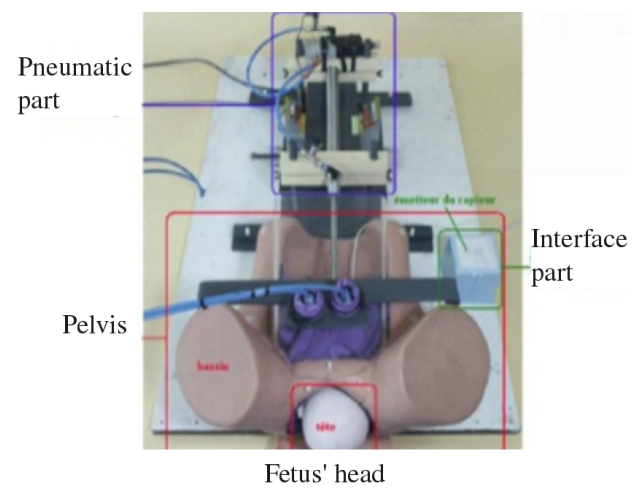

Fig. 2. The BirthSim simulator developed in the Ampere Laboratory [10]

To save computation time, we simplify the anatomy by taking into account only the organs that play a significant role during the delivery. Moreover, we can note that the human body is composed of organs with different non homogeneous mechanical properties. Practically, this highly complicates the computations. Therefore, we made a second simplification by assuming homogenous properties for different anatomical regions.

The fetus is an other complex object. In fact, for the sake of computation time, we cannot model precisely the fetal body. Therefore, we have to find a good approximation of the representation of the fetus. It is interesting to notice that the morphology of a fetus is very different from a grown up child. Indeed, the proportion of the head is very important compared to the body size. Consequently, the essential of the forces applied on the pelvis by the fetus will be caused by the pressure exerted by the head of the fetus. Moreover, we have estimated that the behavior of the head is essentially caused by the skull. That is why we considered the entire fetus having the same mechanical property as the fetal skull. We simplify again this object. After nine months of gestation the bony structure of the fetus is not totally developed [14] and this changes the elastic properties of the skull. So, to simplify the simulation, we apply to our fetus model only an elastic behavior law.

The womb is considered to be a membrane to which we apply two force fields. The first one represents the action of the Uterine Contraction Forces (UCF) that will appear at regular intervals. The second one is the simplification of the effect of the abdominal muscles force. This second forces field is applied to the top of the womb (see Fig. 3) and have to be strong enough to counter the resistance of the pelvis muscle (PMRF). It is important to note that, if the uterus contraction forces help the movement of the fetus, the forces generated by abdominal muscles push the fetus out of the birth canal.

Amniotic liquid is the interface between the fetus, the placenta and the womb. For simplification reason, we modeled it by a very elastic material. 


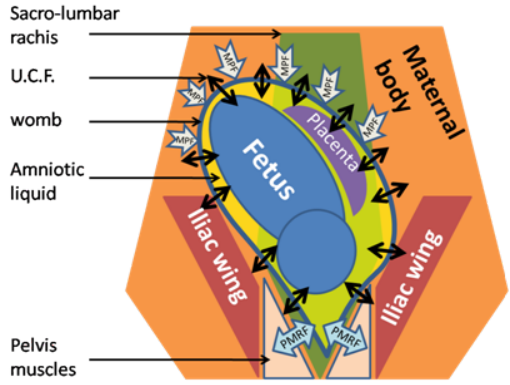

Fig. 3. Anatomic and mechanical pelvis and fetus model scheme

\section{THE GEOMETRICAL MODEL}

For the realization of this biomechanical model, we first need to recover the geometry of the different organs. The segmentation of MRI data provides an over-sampled points cloud which is then converted to a dense mesh representing the geometry of different organs. To decrease the computation time, this mesh should be simplified. The simplification of the mesh size is a sensitive problem. We use ReMESH application [2] to simplify our surface mesh. Indeed, ReMESH suppresses nodes in the regions where the angular gradient is very low, but keeps a representation enough precise to respect the geometry of the object.

Then, the MESH application [1] proposes an efficient method to determine the distance between two surface meshes. Thanks to it, we can estimate the error between the original and the simplified mesh. For sample, we remove more than $90 \%$ of the mesh nodes of the fetus (initial mesh: 45000 nodes; final mesh: 4000 nodes) with a mean error equal to $0.5 \mathrm{~mm}$. The error due to the mesh simplification of the womb, the fetus and the placenta varies from 0.5 to 0.7 $\mathrm{mm}$. Note that, this error margin is highly acceptable for the precision required in our application.

Consequently, the total mesh before the simplification contains more than 1,000,000 tetrahedral elements, and after the ReMESH simplification we have a total number of tetrahedral elements that do not exceed 130, 000 elements.

\section{THE WOMB SIMULATION}

Then, we have to propose a physically based simulation method which simulates the deformation and the force fields. Mass-Spring systems are often used in computer animation, because of their simplicity of implementation and their speed of computation. However, they suffer from very bad stability and the poverty of precision.

On the contrary, Finite Elements Method (FEM) is very stable and precise, with computation time that could be too long for real time simulation. But many solutions have been proposed in the literature to counter this last problem ([6], [7], [12]). Consequently, we chose the FEM for its stability and precision.

One of the most important organs during the delivery is the womb. This muscular part of the female reproductive system is the support of the forces which will push the fetus out of the birth canal. As stated before, two kinds of efforts are applied to fetus: uterine contractions and forces generated by abdominal muscles. During the first stage of the labour, the womb natural contractions push the fetus towards the cervix causing its dilatation. These contractions are regular and uncontrolled. During the second stage, the mother uses her abdominal muscles to apply forces to the uterus to finish the delivery. The action of both types of muscles is necessary to push the fetus out of the mother's body. However, the resultant forces generated by a separate action of the uterus or the abdominal muscles is smaller than the resistance of the pelvis muscles. Consequently, because of the uncontrolled aspect of the womb contractions, it is up to the abdominal forces to be synchronized with theses latter efforts.

Our womb model is composed of four different parts (see Fig. 4): the internal surface, the external superior part, the external surface, and the birth canal.

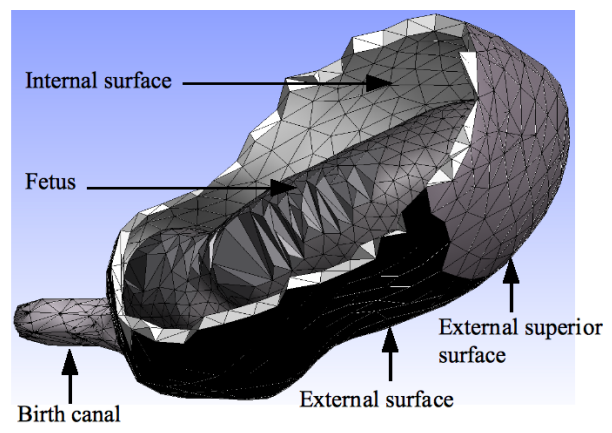

Fig. 4. Different parts of the womb model

The internal surface is used for the contact conditions between the uterus and the fetus. It includes the internal face of the womb and the internal face of vaginal canal. The superior external face is shared by the abdominal muscle and the womb. The second forces field is applied to this part. The womb contraction forces are applied to external surface of the uterus. These efforts are directed towards the birth canal. The birth canal is an extension of the uterus and its role is to guide the fetus as it exits the cervix. Because of the increased volume of the womb, the abdominal muscles are enhanced and their actions can be applied to the upper part of the uterus.

We have developed and compared two models of abdominal efforts. The first model assumes unidirectional forces field, from the top of the uterus toward the centre of the cervix. The second forces model is based on the principle that abdominal muscles cover a more important surface of the uterus and that efforts are applied perpendicularly to the surface of the uterus.

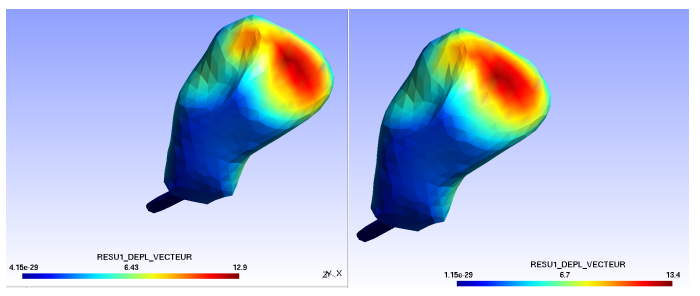

Fig. 5. Comparaison of the displacement field with unidirectionnal force field and normale force field 
We compared the results of deformation and the direction of displacement fields generated by these two forces fields to which we applied to the same womb contractions. The results show that the distribution is slightly more homogeneous in the second case, with efforts following the normal direction (see Fig. 5). Therefore, we only consider abdominal contraction model.

To check the consistency of our model, we integrated the fetus model. The goal is to assess the convergence of the model by observing that the uterus comes into contact with the fetus and placenta meshes. At this step, for the simplification of the computation, we assume the fetus rigid by increasing its elastic modulus. In these new conditions, we obtain a good deformation of the womb geometry, with satisfying contact conditions between different organs.

Next we study the displacement of the fetus in the two stages of the delivery. At the first stage, we only apply the uterus contraction and we want to check, if we have a good positioning of the fetus. We observe a very light descent $(1 \mathrm{~mm})$ with a rotation of the fetus' body. At the second stage of the delivery, we add the abdominal forces to verify if we have a correct displacement of the fetus in the direction of the cervix. We observe a good descent $(20-22 \mathrm{~mm})$ of the fetus near to the cervix (see Fig. 6).

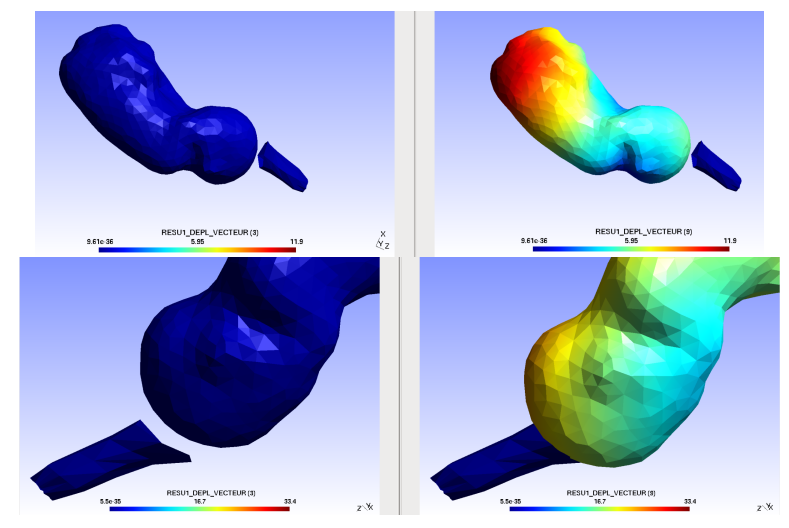

Fig. 6. Fetus displacement with uterus contraction forces (up) or uterus contraction and abdominal forces (down)

\section{CONCLUSION}

We proposed a biomechanical model based on the Finite Elements Method and mechanical laws to simulate the delivery and to recover the different forces applied to the different organs concerned by childbirth. Our model takes into account the principal obstetrical organs. We verified that the different simplifications that we apply (like using an elastic behaviour law, assuming homogeneous elastic mechanical properties for the fetus, or the simplification of the force fields applied to our model) do not result in erroneous behaviors. We used the Finite Elements Method to obtain dynamic simulations and to estimate different mechanical parameters such as forces, displacements to be input to BirthSim childbirth simulator. At this stage, our goal was to model a realistic anatomical behaviour of the organs, by finding the right boundary conditions for each organ. Interactive time performance was not researched. Moreover, we propose a simplified model to verify the feasibility of our approach. In the future, we will develop a more precise model, using more realistic contraction forces (several elementary contractions). Then, the next step will be devoted to accelerate the computation time, by introducing new real time simulation algorithms.

\section{ACKNOWLEDGMENT}

This work is partly financed by a grant of the GMCAO project of the cluster ISLE of the Rhône-Alpes region. Special thanks to Jeremy Arquez (TELECOM ParisTech, CNRS, UMR-5141, LTCI) for the segmentation of the medical data provide by the Prof. C. Adamsbaum (St Vincent de Paul hospital, Paris).

\section{REFERENCES}

[1] N. Aspert, D. Santa-Cruz, and T. Ebrahimi. MESH: Measuring errors between surfaces using the hausdorff distance. In IEEE International Conference on Multimedia and Expo, volume I, pages 705 - 708, 2002.

[2] M. Attene and B. Falcidieno. ReMESH: An interactive environment to edit and repair triangle meshes. In Shape Modeling and Applications (SMI), pages 271-276, 2006.

[3] R. Baumann, W. Maeder, G. Glauser, and R. Clavel. The pantoscope: A spherical remote-center-of-motion parallel manipulator for force reflexion. In IEEE International Conference on Robotic and Automation, Albuquerque, Etats Unis, 1997.

[4] Jean-Daniel Boissonnat and Bernhard Geiger. 3d simulation of delivery. In G. M. Nielson and D. Bergeron, editors, Visualization 93, pages 416-419, San Jose CA, 1993. IEEE Computer Society Press.

[5] H. K. Cakmak. Advanced surgical training in laparoscopy with vest simulators. In 2eme Worshop on Basic Anatomy and advanced Technology in Laparoscopic Suregery, Kiel Allemagne, 2003.

[6] S. Cotin, H. Delingette, J.-M Clement, V. Tasseti, J. Marescaux, and N. Ayache. Volumetric deformable models for simulation of laparoscopic surgery. In International Symposium on Computer and communication Systems for Image Guided Diagnosis and Therapy, Computer Assisted Radiology, Paris, France, 1996.

[7] G. Debunne, M. Desbrun, A. Barr, and M.-P Cani. Interactive multi resolution animation of deformable models. In Eurographics, Worshop on Computer Animation and Simulation, pages 133-144, 1999.

[8] P. Dubois, J.-F Rouland, P. Meseure, S. Karpf, and C. Chaillou. Simulator for laser photocoagulation in ophtalmology. IEEE Transaction in Biomedical Engineering, 42(7), 1995.

[9] A. Kheddar, C. Devine, M. Brunel, C. Duriez, and O. Sidony. Preliminary design of a childbirth simulator haptic feedback. In IEEE/RSJ, International Conference on Inteligent Robots and Systems, volume 4, pages 3270-3275, 2004.

[10] R. Moreau, M.-T Pham, R. Silveira, T. Redarce, X. Brun, and O.Dupuis. Design of a new instrumented forceps: Application to safe obstetrical forceps blade placement. IEEE Transactions on Biomedical Engineering, 7(54), july 2007.

[11] R.-J Muffler. Av-8b harrier ii training capabilities. In AIAA Flight Simulator Technologies Conference, pages 11-15, St Louis, MO, USA, 1985.

[12] M. Nesme, Y. Payan, and F. Faure. Efficient, physically plausible finite elements. In John Dingliana and Fabio Ganovelli, editors, Eurographics 2005, Short papers, August, 2005, Trinity College, Dublin, Irlande, 2005.

[13] R. Riener and R. Burgkart. Birth simulator (geburtensimulator), 2003.

[14] J.-P Schall, D. Riethmuller, R. Maillet, and M. Uzan. Mécanique et Technique Obstétricales. sauramps medical, troisième edition, février 2007.

[15] R. Silveira, M.-T Pham, T. Redarce, M. Btemps, and O. Dupuis. A new mechanical birth simulator: Birthsim. In IEEE/RSJ International Conference on Intelligent Robots and Systems (IROS04), pages 39483954, Sendai, Japan, 2004.

[16] P.-Y Zambelli, C. Bregand, S. Dewarrat, G. Marti, C. Baur, and P. Leyvraz. planning and navigation solution in resurfacing hips surgery : A way to reduce the surgical approach. In Poster session, 3rd Annual meeting of the International Society Orthopaedic Surgery, Marbella Espagne, 2003. 